\title{
Epidemiology of Cercospora Leaf Spot on Sugar Beet: Modeling Disease Dynamics Within and Between Individual Plants
}

\author{
J. Vereijssen, J. H. M. Schneider, and M. J. Jeger
}

First and second authors: IRS (Institute of Sugar Beet Research), P.O. Box 32, 4600 AA, Bergen op Zoom, The Netherlands; third author:

Division of Biology, Faculty of Life Sciences, Imperial College London, Wye Campus, Wye, Ashford, Kent, TN25 5AH, UK. Accepted for publication 19 July 2007.

\begin{abstract}
Vereijssen, J., Schneider, J. H. M., and Jeger, M. J. 2007. Epidemiology of Cercospora leaf spot on sugar beet: Modeling disease dynamics within and between individual plants. Phytopathology 97:1550-1557.

Disease dynamics of Cercospora leaf spot (CLS) of sugar beet was analyzed at two hierarchical scales: as vertical profiles within individual plants and in relation to disease on neighboring plants. The relative contribution of different leaf layers to increase in CLS was analyzed using a simple continuous-time model. The model was fitted to data from two field trials in the Netherlands: one in an area with a long history of CLS, the other in an area where CLS has only recently established; in each case these were unsprayed and twice-sprayed treatments. There were differences in the relative contribution of different leaf layers to disease increase on the target leaf layer according to the CLS history and whether

increase at the target leaf layer was significant. Thus, CLS increase at the target leaf layer was determined mainly by disease severity at that leaf layer and to a lesser extent by disease at the lower leaf layer. Our continuous-time model was also used to analyze CLS increase on an individual sugar beet plant in relation to its own and its neighbor's level of disease in field trials at five locations in the two CLS areas over two years. In all field trials, the contribution of the target plant itself to disease increase (auto-infection) was larger than that of its neighboring plants (allo-infection). The overall analysis in the two CLS areas also indicated a larger contribution of the target plant to its disease increase than of neighboring plants, and this pattern was also apparent in a pooled analysis across all sites. Thus, CLS increase on a sugar beet plant was mainly determined by the disease severity on that plant and to a lesser extent by its within-row neighboring plants.
\end{abstract} the plants were sprayed or unsprayed. In both field trials, parameter estimates giving the relative contribution of the target leaf layer to disease increase at that leaf layer were higher than those for the lower leaf layer. On only a few occasions the contribution of an upper leaf layer to disease
Additional keywords: Beta vulgaris, between plant disease dynamics, Cercospora beticola, within-plant disease dynamics.
Cercospora leaf spot (CLS), caused by the polycyclic pathogen Cercospora beticola Sacc., is economically important in many beet growing regions of the world. CLS can cause a reduction in gross sugar yield of up to $42 \%$ (15). In the Netherlands two CLS areas can be distinguished. In the southeastern part of the country (provinces of Limburg and southeastern Noord-Brabant) there has been a long CLS history with mostly high disease severities (HDS area). In the remaining sugar beet growing areas (e.g., province of Gelderland and Drenthe) occurrences of CLS have been more recent, since 1994, with mostly low disease severities (LDS area). This study was motivated by preliminary observations in 1999, where a difference in CLS occurrence between the two areas was noted. We use the HDS and LDS terminology as shorthand throughout this paper, whilst recognizing that it is confounded with area. In the HDS areas, a uniform CLS pattern occurred, where almost all plants were diseased with about the same level of disease, assessed according to the Agronomica disease severity index $(1,17)$. In the LDS areas, there was a patchy occurrence of CLS with healthy plants occurring next to severely diseased plants. We questioned whether there were differences in the pattern of disease progress between the two areas at different hierarchical levels. A geostatistical approach was used to characterize the spatial disease pattern of CLS (19), but this did not take into account the temporal increase in disease over the growing season. Previously, authors have used disease progress curves to elucidate

Corresponding author: J. H. M. Schneider; E-mail address: schneider@irs.nl

doi:10.1094/PHYTO-97-12-1550

(c) 2007 The American Phytopathological Society disease epidemiology (12), evaluate cultivar resistance (10), represent output from simulation models (13), and assess integrated management systems $(13,16,23)$. Few studies have partitioned the effects on disease progress curves due to inoculum derived from and infecting a given plant (auto-infection) and inoculum derived from neighboring plants (allo-infection). Recently Willocquet and Savary (21) presented an epidemiological model integrating three hierarchical scales (site within-leaf, leaf, and plant) and simulated disease dynamics under different auto- and allo-infection ratios.

Dispersal of a pathogen from a diseased plant or diseased leaf can be horizontal from plant to plant, leading to the development of disease gradients along rows within fields, but also vertical, within a plant, from older to younger leaves (22). The pathogen is dispersed over short distances $(7,19)$; the elongated conidia with smooth surfaces and thin hyaline walls are characteristic for splash-dispersed spores (3). During rain, conidia are splashed in all directions from sporulating lesions on infected leaves to younger leaves by raindrops, and downwards to older leaves by droplet runoff from foliage. Conidia may also splash horizontally to neighboring plants. Initial infections arise from conidia dispersed from infected leaf debris at the soil surface $(7,9,22)$. These initial infections could result in severe epidemics developing under optimal environmental conditions, and the presence of infected debris may differ in areas with long-established or only recent CLS histories.

The first objective in this study was to determine whether there was any relationship between the vertical profile of disease on a plant and the within-plant increase of CLS. We determined the vertical profile of disease on an individual leaf basis and used a simple model to estimate the contribution of different leaf layers 
to disease increase on a target leaf layer. We addressed a second related question; whether the pattern of within-plant disease increase was affected by fungicide application.

The second objective was to determine whether there was any relationship between disease increase on a target plant and the level of disease on that plant and on neighboring plants. We used the same model to obtain parameter estimates reflecting the contributions made to disease increase on a target plant by that plant and its neighbors. We addressed two questions: firstly, whether there were differences in parameter estimates within a field trial over the two years, and secondly, whether there were differences between the two areas with respect to the estimated parameters.

\section{MATERIALS AND METHODS}

Model Development. In tackling the objectives we developed a simple continuous-time model of disease dynamics, appropriate for both hierarchical scales between leaf layers within a plant, and between plants within a row. We stress that the model is not intended to represent in detail, the known epidemiology of CLS, but rather to be used as a calculation tool to disentangle the various sources of inoculum and their contribution to disease dynamics at different hierarchical scales. As such, it can be used for other polycyclic foliar diseases. We define the rate of change in disease at one of these scales as

$$
\frac{d Y_{i}}{d t}=b S\left(M-Y_{i}\right)
$$

where $Y_{i}$ is disease severity on the target leaf layer or individual plant (denoted by $i$, the spatial location either within a plant or within a row), $S$ is a measure of the inoculum produced by diseased tissue at a leaf layer or on an individual plant, $b$ is a rate constant, and $M$ is an upper asymptote for severity. The units of $b$ depend on the units subsequently defined for $S$. We then specify an expression for $S$, the amount of inoculum, to develop the model further. We assume that the rate of change in disease on a defined leaf layer or individual plant is a sufficient measure of subsequent inoculum production. We assume that the mean latent period to be approximately the same as the time interval between assessments and that spore production is greatest immediately following the latent period, as shown conceptually in Figure 1.

Latent period depends on temperature and will vary with time but for most polycyclic pathogens, overlapping infection cycles soon occur as an epidemic develops leading to a complex age structure among lesions. On this basis we assume that the disease assessment interval is not critical in applying the model. Similarly, spore production depends on environmental conditions and thus the conceptual representation in Figure 1 is more realistic than that of a constant spore production. We also recognize that for diseases such as CLS, toxin-induced necrosis may affect the assumed relationship between spore production and disease severity, and this may lead to systematic biases in applying the model.

We partition the product $b S$ into the linear sum

$$
b S \approx b_{i} \frac{d Y_{i, t-1}}{d t}+b_{i-1} \frac{d Y_{i-1, t-1}}{d t}+b_{i+1} \frac{d Y_{j+1, t-1}}{d t}
$$

where $b$ is now a location-specific parameter: $i$ refers to the target leaf layer or individual plant, $i-1$ refers to the leaf layer below or the neighboring plant down the row, and $i+1$ to the leaf layer above or the neighboring plant up the row; and $t-1$ refers to the time of the previous disease assessment $(\Delta t=1)$.

Substitution of equation 2 into 1 , followed by re-arrangement and integration, gives the model

$$
-\ln \left(M-Y_{i, t}\right)=b_{i} Y_{i, t-1}+b_{i-1} Y_{i-1, t-1}+b_{i+1} Y_{i+1, t-1}
$$

Estimates of $b_{i}, b_{i-1}$ and $b_{i+1}$ and the intercept were then obtained by least squares regression, which also gave an estimate of the intercept with $Y_{i, t-1}=Y_{i-1, t-1}=Y_{i+1, t-1}=0$. Note that the $b$ coefficients are dimensionless. Although the $b$ coefficient is likely to vary with time, we use the partitioning above to enable a comparison of the relative contribution of each defined leaf layer or individual plant to disease dynamics over the time course of the epidemic. Models were fitted using GenStat 5 (Lawes Agricultural Trust, Rothamsted Research, UK).

Vertical profiles of CLS on individual sugar beet plants: Field trial establishment. In 1999, trials were established in an HDS area at Maasbree where CLS has been established since at least 1973, with severe epidemics occurring (mean leaf area diseased $>10 \%$ ), and in an LDS area at Toldijk in the province of Gelderland, where CLS occurrence has only been recent and epidemics typically have mean leaf area diseased $<10 \%$. Both trials were established in fields with sandy soils, and were established as part of a larger study on supervised management of CLS (18). Farmers' fields were drilled with sugar beet cv. Tiara at Maasbree (sown 10 April) and cv. Rebecca at Toldijk (sown 29 March), both cultivars being equally susceptible for CLS. The experimental design consisted of 6 treatments in 4 randomized blocks, although only plots that were unsprayed or sprayed twice were used for the data analyzed in this study. A systemic triazole fungicide (Score 250EC, a.i., difenoconazole $250 \mathrm{~g} / \mathrm{liter}$ ) (Syngenta, The Netherlands) was applied ( 0.41 per ha at 3.3 bar) at both locations using a hand-held horizontal spray boom with six Teejet 11003 versus nozzles $50 \mathrm{~cm}$ apart with 50 mesh anti-drop screen at a walking speed of 1 meter per second. Fungicide treatments were applied twice at Maasbree on 30 July and 11 August, and at Toldijk on 13 and 31 August. Experimental units were $3 \mathrm{~m}$ ( 6 rows of sugar beet) $\times 12.5 \mathrm{~m}$ and blocks were separated by $2.5 \mathrm{~m}$ bare soil.

At Maasbree, plants were monitored weekly from 27 July to 13 September, and at Toldijk from 29 July to 23 September. For each experimental unit (plot) of $3 \mathrm{~m} \times 12.5 \mathrm{~m}$, five plants (three in row 2 and two in row 5) (20 plants per treatment) were used for disease severity assessments. All monitored plants in a row were separated by one plant. Disease severity was assessed for each leaf of each plant at predefined levels of leaf area diseased $(0,0.1$, $0.3,0.5,1,5,10,25,50,75,90$, and $100 \%)(17)$. The first three green petioles were tagged with different colored rings, thus enabled monitoring of disease increase on individual leaves throughout the season. As the first green leaf died, rings were moved anticlockwise following the rosette until the next green petiole was reached.

Estimation of parameters. Each plant was divided into leaf layers of 5 leaves each, and the mean disease severity at time $t$ $\left(Y_{i}\right)$ for each leaf layer was calculated. Once 15 leaves were present regression analyses could be undertaken and were repeated with the formation of successive leaf layers. The dependent

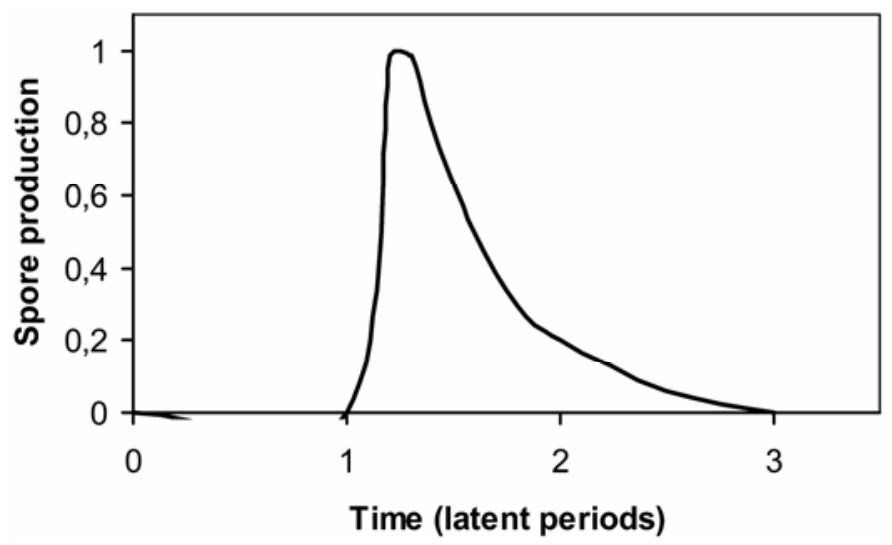

Fig. 1. Conceptual representation of spore production (undefined native units). Spore production is greatest immediately following the latent period. 
variate (the left side of equation 3) was calculated directly for each leaf layer, with $M=100 \%$. The independent variates are disease severity on the defined leaf layer $(i)$, and on leaf layers above $(i+1)$ and below $(i-1)$, at the preceding time $t-1$. Regression analyses were made for Toldijk and Maasbree separately due to the different cultivars, planting dates, and fungicide application timings at the two sites. Only general qualitative comparisons of parameter estimates were made between sites. A priori parameter estimates were not allowed to take negative values, and where this happened the variate was omitted from the model.

The contribution of within-row neighboring plants to disease dynamics: Field trial establishment. Disease severities were assessed every 1 to 2 weeks from sugar beet fields in the Netherlands in 2000 and 2001 as described by Vereijssen et al. (19). Disease severity was assessed visually on a whole plant basis using the pictorial Agronomica disease severity index (17), covering a scale from 0 (healthy) to 5 (totally destroyed foliage) the maximum value. Two field trials, at Vlodrop (V) in the HDS area and Toldijk1 (T1) in the LDS area, were selected for detailed assessments in 2000 because of preliminary observations made in these two areas in 1999. These fields were redrilled with sugar beet in 2001 and assessments were repeated. In 2000, a field trial consisted of 30 rows $\times 7.5 \mathrm{~m}$ with 30 plants assessed in each row, whereas in 2001 all plants were assessed in each of the 30 rows. Spacing between rows was constant at $0.50 \mathrm{~m}$. The three rows closest to the edge of a field were ignored. Farmers' fields were drilled with different cultivars equally susceptible to CLS at each location. The field trials were unsprayed allowing a natural CLS epidemic to develop. Additional data were collected in an HDS area at Koningsbosch (province of Limburg) in 2000 and 2001 and in an LDS area at Wehl (W) and a second field at Toldijk2 (T2), both in the province of Gelderland, in 2000 only. The number of plants in the field trials ranged between 717 and 980 . Disease assessment took place every week in 2000, and every 2 weeks in 2001.

Estimation of parameters. The dependent variable (left hand side of equation 2) was calculated directly for each plant at each assessment. We substitute the index $j$ for $i$ here and in the Results, to distinguish the within-row and within-plant scales. Any severity values greater than 5 (an artifact of the Agronomica disease severity index) and missing values were excluded. The independent variates are disease severity on the defined plant $(j)$, and on neighboring plants $(j+1, j-1)$ within rows, at the preceding time $t-1$. Previously (19), we found no significant acrossrow effects. Assuming no directional bias within rows in inoculum dispersal, the mean of $Y_{-1}$ and $Y_{+1}$ at time $t-1$ was taken. Regression analyses were made for Vlodrop and Toldijk separately to test for differences in parameter estimates between field trials or areas and between years within a field trial. If the analysis showed no significant differences in the parameter esti- mates, an overall regression was conducted. A priori, parameter estimates were not allowed to take negative values, and if this occurred, the corresponding independent variable was omitted from the model.

\section{RESULTS}

Vertical profiles of CLS on individual sugar beet plants. Disease severity at Maasbree (HDS) was, as expected, more severe than at Toldijk (LDS). In the unsprayed treatment the mean leaf area diseased was $13 \%$ at Maasbree and $4 \%$ at Toldijk. For the twice-sprayed treatment the mean leaf area diseased was $0.9 \%$ at Maasbree and $1.3 \%$ at Toldijk (data not shown). The increase in disease severity per leaf layer over time of a model plant (average of six plants) at Maasbree for the unsprayed and sprayed treatment is illustrated in Figure 2. In total some seven leaf layers (35 leaves) were considered for each plant over the period of assessment (Fig. 2). It is striking that only leaf layers two, three, and four (each containing five leaves) have a fast increase in mean disease severity in the unsprayed treatment (Fig. 2). In the sprayed treatment, increase in disease on these leaf layers is much less, but they are still the ones with highest disease severity (Fig. 2).

In both the HDS and LDS fields, under-sprayed and unsprayed conditions, the model gave coefficients of determination $\left(R^{2}\right)$ between 57.6 and $97.0 \%$ (Tables 1 to 4 ), and in most cases more than $80 \%$. Coefficients are only entered for the respective leaf layers in Tables 1 through 4 when they were positive and significant $(P<0.05)$. The back-transformed values of the intercept estimates were close to zero in all cases.

LDS (Toldijk) versus HDS (Maasbree) unsprayed (Tables 1 and 2). In both areas the parameter estimates for the target leaf layer were, with few exceptions, higher than those for the lower leaf layer. The parameter estimates for the target leaf layer were consistently greater at Maasbree $(0.009$ to 0.035 ; median $=0.020)$ (Table 1$)$ than at Toldijk (0.009 to 0.024 ; median $=0.016)$ (Table 2 ). This indicates that disease increase on the target leaf layer per unit of disease was more influenced by disease on this leaf in the HDS field. At Toldijk, parameter estimates for the regression coefficient of the lower leaf layer were more often significant in describing disease increase on the target leaf layer than at Maasbree, and the parameter estimates were higher (0.003 to 0.050 ; median $=0.009)($ Table 2$)$ compared with Maasbree $(0.002$ to 0.003 ; median $=0.002)$ (Table 1$)$ respectively. This indicated that the lower leaf layer contributed more per unit of disease to disease increase of the target leaf layer in the LDS than in the HDS field. Disease severity on the upper leaf layer made a significant contribution to the model only in unsprayed plots for 8 out of 20 plants $(0.002$ to 0.010 ; median $=0.006)$ at Toldijk (LDS field) (Table 2). When both upper and lower leaf layers were included in the model at Toldijk, the parameter estimates for the
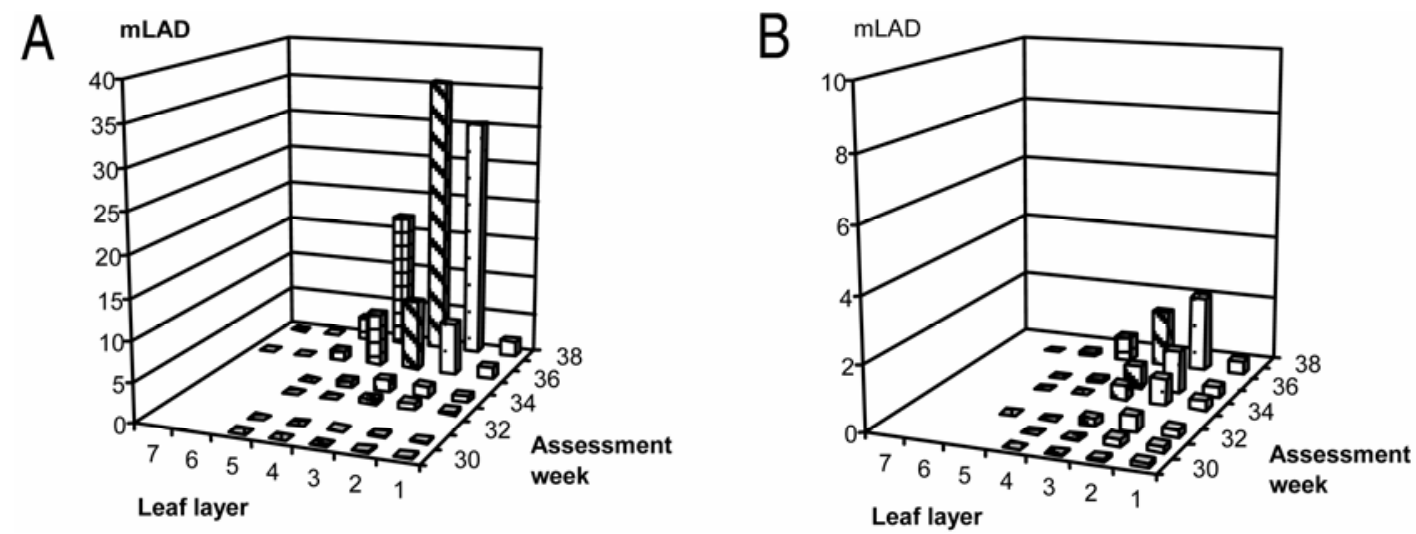

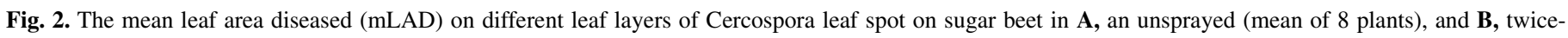
sprayed situation (mean of six plants) with a triazole fungicide. 
upper layer were of roughly the same magnitude as for the lower layer, but both were always smaller than of the target leaf layer (Table 2). The upper leaf layer did not make a significant contribution to disease increase at Maasbree and is omitted from Table 1.
LDS (Toldijk) versus HDS (Maasbree) sprayed (Tables 3 and 4). As with the unsprayed treatment, parameter estimates for the target leaf layer were always higher than those for the lower leaf layer at both sites. The range of parameter estimates for the target leaf layer were similar for both areas (Maasbree: 0.008 to

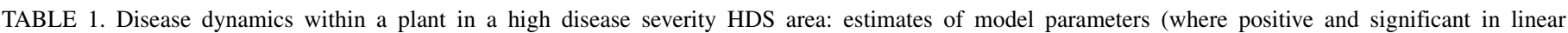

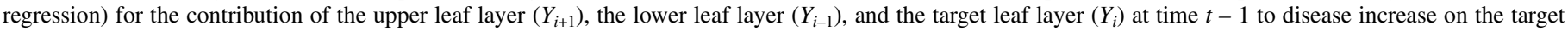
leaf layer at time $t$ in the unsprayed treatment at Maasbree

\begin{tabular}{|c|c|c|c|c|c|c|c|c|}
\hline Plant & $Y_{i-1, t-1}$ & $\mathrm{SE}^{\mathrm{a}}$ & $P$ value ${ }^{\mathrm{b}}$ & $Y_{i, t-1}$ & SE & $P$ value & $R^{2}$ & $N^{\mathrm{c}}$ \\
\hline 1 & & & & 0.023 & 0.00082 & $<0.001$ & 90.0 & 85 \\
\hline 2 & & & & 0.034 & 0.00125 & $<0.001$ & 90.3 & 78 \\
\hline 3 & & & & 0.028 & 0.00126 & $<0.001$ & 89.4 & 56 \\
\hline 6 & & & & 0.022 & 0.00113 & $<0.001$ & 79.3 & 100 \\
\hline 7 & 0.003 & 0.00155 & 0.035 & 0.022 & 0.00155 & $<0.001$ & 80.9 & 96 \\
\hline 11 & & & & 0.015 & 0.00047 & $<0.001$ & 92.5 & 85 \\
\hline 12 & & & & 0.014 & 0.00040 & $<0.001$ & 92.4 & 100 \\
\hline 13 & 0.002 & 0.00067 & 0.011 & 0.012 & 0.00067 & $<0.001$ & 91.4 & 76 \\
\hline 16 & & & & 0.013 & 0.00048 & $<0.001$ & 90.6 & 81 \\
\hline 17 & & & & 0.014 & 0.00050 & $<0.001$ & 91.0 & 80 \\
\hline 21 & & & & 0.011 & 0.00052 & $<0.001$ & 85.9 & 71 \\
\hline 22 & & & & 0.011 & 0.00047 & $<0.001$ & 86.3 & 85 \\
\hline 23 & 0.002 & 0.00073 & 0.005 & 0.009 & 0.00073 & $<0.001$ & 90.2 & 77 \\
\hline 26 & 0.002 & 0.00071 & 0.019 & 0.010 & 0.00071 & $<0.001$ & 89.8 & 76 \\
\hline 27 & & & & 0.019 & 0.00042 & $<0.001$ & 95.2 & 99 \\
\hline 31 & & & & 0.035 & 0.00110 & $<0.001$ & 92.3 & 81 \\
\hline 32 & & & & 0.020 & 0.00088 & $<0.001$ & 86.9 & 77 \\
\hline 33 & & & & 0.033 & 0.00120 & $<0.001$ & 90.4 & 80 \\
\hline 36 & & & & 0.024 & 0.00089 & $<0.001$ & 91.0 & 72 \\
\hline 37 & & & & 0.025 & 0.00079 & $<0.001$ & 92.6 & 79 \\
\hline Minimum $^{\mathrm{d}}$ & 0.002 & & & 0.009 & & & & \\
\hline Maximum $^{\mathrm{e}}$ & 0.003 & & & 0.035 & & & & \\
\hline Median $^{f}$ & 0.002 & & & 0.020 & & & & \\
\hline
\end{tabular}

a Standard error of the parameter estimate.

${ }^{b}$ Level of significance.

c Number of target leaf layers at position $i$.

d Minimum value of the parameter estimate.

e Maximum value of the parameter estimate.

${ }^{f}$ Median value of parameter estimate.

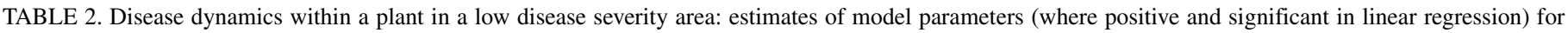

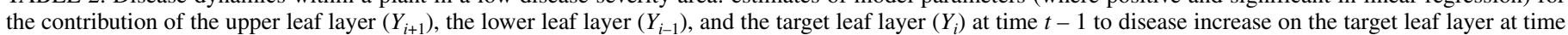
$t$ in the unsprayed treatment at Toldijk

\begin{tabular}{|c|c|c|c|c|c|c|c|c|c|c|c|}
\hline Plant & $Y_{i+1, t-1}$ & $\mathrm{SE}^{\mathrm{a}}$ & $P$ value ${ }^{\mathrm{b}}$ & $Y_{i-1, t-1}$ & SE & $P$ value & $Y_{i, t-1}$ & SE & $P$ value & $R^{2}$ & $N^{\mathrm{c}}$ \\
\hline 1 & 0.010 & 0.00307 & 0.002 & 0.016 & 0.00307 & $<0.001$ & 0.015 & 0.00402 & $<0.001$ & 80.0 & 98 \\
\hline 2 & & & & 0.011 & 0.00111 & $<0.001$ & 0.011 & 0.00111 & $<0.001$ & 91.5 & 78 \\
\hline 3 & & & & & & & 0.024 & 0.00075 & $<0.001$ & 90.6 & 105 \\
\hline 6 & & & & 0.050 & 0.00448 & $<0.001$ & & & & 57.6 & 91 \\
\hline 7 & & & & 0.022 & 0.00454 & $<0.001$ & 0.016 & 0.00454 & $<0.001$ & 64.9 & 82 \\
\hline 11 & & & & 0.004 & 0.00083 & $<0.001$ & 0.016 & 0.00083 & $<0.001$ & 90.1 & 103 \\
\hline 12 & 0.002 & 0.000801 & 0.005 & 0.005 & 0.00080 & $<0.001$ & 0.012 & 0.00107 & $<0.001$ & 93.2 & 75 \\
\hline 13 & & & & 0.009 & 0.00093 & $<0.001$ & 0.016 & 0.00093 & $<0.001$ & 93.0 & 90 \\
\hline 16 & & & & 0.009 & 0.00225 & $<0.001$ & 0.023 & 0.00225 & $<0.001$ & 84.1 & 103 \\
\hline 17 & 0.006 & 0.00176 & 0.002 & 0.004 & 0.00176 & 0.031 & 0.018 & 0.00244 & $<0.001$ & 88.2 & 84 \\
\hline 21 & 0.009 & 0.00359 & 0.014 & 0.009 & 0.00359 & 0.01 & 0.014 & 0.00469 & 0.004 & 64.2 & 87 \\
\hline 22 & 0.008 & 0.00194 & $<0.001$ & 0.006 & 0.00194 & 0.003 & 0.009 & 0.00249 & $<0.001$ & 70.3 & 81 \\
\hline 23 & 0.005 & 0.0013 & $<0.001$ & 0.003 & 0.00130 & 0.024 & 0.016 & 0.00159 & $<0.001$ & 87.2 & 78 \\
\hline 26 & & & & 0.010 & 0.00255 & $<0.001$ & 0.015 & 0.00255 & $<0.001$ & 67.0 & 87 \\
\hline 27 & & & & 0.007 & 0.00171 & $<0.001$ & 0.021 & 0.00171 & $<0.001$ & 83.1 & 87 \\
\hline 31 & 0.005 & 0.00168 & 0.002 & & & & 0.013 & 0.00168 & $<0.001$ & 61.0 & 87 \\
\hline 32 & & & & 0.007 & 0.00130 & $<0.001$ & 0.018 & 0.00130 & $<0.001$ & 91.3 & 70 \\
\hline 33 & & & & 0.010 & 0.00153 & $<0.001$ & 0.018 & 0.00153 & $<0.001$ & 87.7 & 72 \\
\hline 36 & & & & 0.010 & 0.00156 & $<0.001$ & 0.015 & 0.00156 & $<0.001$ & 86.3 & 87 \\
\hline 37 & 0.004 & 0.00099 & $<0.001$ & 0.006 & 0.00099 & $<0.001$ & 0.013 & 0.00121 & $<0.001$ & 90.0 & 84 \\
\hline Minimum $^{\mathrm{d}}$ & 0.002 & & & 0.003 & & & 0.009 & & & & \\
\hline Maximum $^{\mathrm{e}}$ & 0.010 & & & 0.050 & & & 0.024 & & & & \\
\hline Median $^{\mathrm{f}}$ & 0.006 & & & 0.009 & & & 0.016 & & & & \\
\hline
\end{tabular}

a Standard error of the parameter estimate.

${ }^{b}$ Level of significance.

c Number of target leaf layers at position $i$.

${ }^{\mathrm{d}}$ Minimum value of the parameter estimate.

e Maximum value of the parameter estimate.

${ }^{\mathrm{f}}$ Median value of parameter estimate. 
0.014, median $=0.011$; Toldijk: 0.009 to 0.017 , median $=0.012$ ) . The range of parameter estimates for the regression coefficient of the lower leaf layer at Maasbree $(0.002$ to 0.004 ; median $=0.003)$ (Table 3) were similar to those for Toldijk (0.001 to 0.003 ; median $=0.002)($ Table 4$)$. In contrast to the unsprayed treatment, the pattern of disease increase within the sprayed treatment appeared similar for the HDS and LDS trials.

Sprayed versus unsprayed at HDS (Maasbree) (Tables 1 and 3). With a fungicide treatment, the regression coefficients of the lower leaf layer were more often significant in explaining

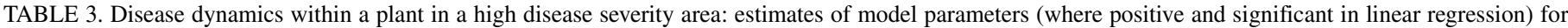

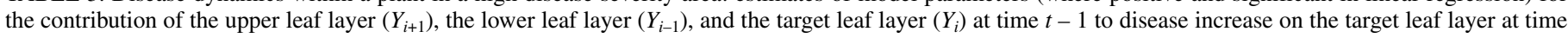
$t$ in the twice-sprayed treatment at Maasbree

\begin{tabular}{|c|c|c|c|c|c|c|c|c|c|c|c|}
\hline Plant & $Y_{i+1, t-1}$ & $\mathrm{SE}^{\mathrm{a}}$ & $P$ value $^{\mathrm{b}}$ & $Y_{i-1, \mathrm{t}-1}$ & SE & $P$ value & $Y_{i, t-1}$ & $\mathrm{SE}$ & $P$ value & $R^{2}$ & $N^{\mathrm{c}}$ \\
\hline 1 & & & & & & & 0.013 & 0.00039 & $<0.001$ & 94.9 & 58 \\
\hline 2 & 0.002 & 0.000783 & 0.005 & 0.003 & 0.00079 & $<0.001$ & 0.011 & 0.00103 & $<0.001$ & 91.3 & 61 \\
\hline 3 & & & & 0.002 & 0.00058 & $<0.001$ & 0.010 & 0.00058 & $<0.001$ & 92.3 & 76 \\
\hline 6 & & & & 0.002 & 0.00066 & $<0.001$ & 0.010 & 0.00066 & $<0.001$ & 84.8 & 68 \\
\hline 7 & & & & & & & 0.012 & 0.00054 & $<0.001$ & 87.1 & 73 \\
\hline 11 & & & & 0.004 & 0.00083 & $<0.001$ & 0.011 & 0.00083 & $<0.001$ & 91.3 & 64 \\
\hline 12 & & & & & & & 0.011 & 0.00081 & $<0.001$ & 68.8 & 78 \\
\hline 13 & & & & 0.004 & 0.00063 & $<0.001$ & 0.009 & 0.00063 & $<0.001$ & 93.4 & 70 \\
\hline 16 & & & & 0.002 & 0.00060 & $<0.001$ & 0.011 & 0.00060 & $<0.001$ & 93.2 & 74 \\
\hline 17 & & & & 0.004 & 0.00098 & $<0.001$ & 0.010 & 0.00098 & $<0.001$ & 87.3 & 75 \\
\hline 21 & & & & & & & 0.013 & 0.00053 & $<0.001$ & 88.7 & 76 \\
\hline 22 & & & & & & & 0.014 & 0.00048 & $<0.001$ & 91.2 & 78 \\
\hline 23 & & & & & & & 0.014 & 0.00036 & $<0.001$ & 94.8 & 75 \\
\hline 26 & & & & & & & 0.013 & 0.00042 & $<0.001$ & 93.4 & 63 \\
\hline 27 & & & & 0.004 & 0.00080 & $<0.001$ & 0.009 & 0.00080 & $<0.001$ & 89.8 & 74 \\
\hline 31 & & & & 0.003 & 0.00078 & $<0.001$ & 0.009 & 0.00078 & $<0.001$ & 86.5 & 67 \\
\hline 32 & & & & 0.004 & 0.00088 & $<0.001$ & 0.008 & 0.00088 & $<0.001$ & 86.8 & 72 \\
\hline 33 & & & & 0.003 & 0.00069 & $<0.001$ & 0.010 & 0.00069 & $<0.001$ & 83.9 & 68 \\
\hline 36 & & & & 0.002 & 0.00074 & $<0.001$ & 0.009 & 0.00074 & $<0.001$ & 88.5 & 68 \\
\hline 37 & & & & 0.002 & 0.00057 & $<0.001$ & 0.011 & 0.00057 & $<0.001$ & 92.5 & 71 \\
\hline Minimum $^{\mathrm{d}}$ & 0.002 & & & 0.002 & & & 0.009 & & & & \\
\hline Maximum $^{\mathrm{e}}$ & 0.002 & & & 0.004 & & & 0.014 & & & & \\
\hline Median $^{\mathrm{f}}$ & 0.002 & & & 0.003 & & & 0.011 & & & & \\
\hline
\end{tabular}

a Standard error of the parameter estimate.

$\mathrm{b}$ Level of significance.

c Number of target leaf layers at position $i$.

${ }^{\mathrm{d}}$ Minimum value of the parameter estimate.

e Maximum value of the parameter estimate.

${ }^{\mathrm{f}}$ Median value of parameter estimate.

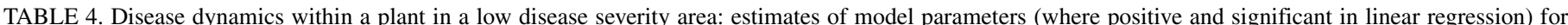

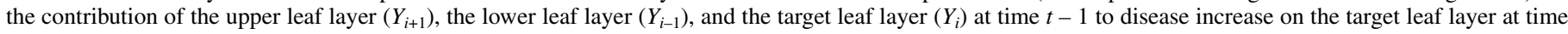
$t$ in the twice-sprayed treatment at Toldijk $-\ln \left(100-Y_{i, t}\right)=b_{i} Y_{i, t-1}+b_{i-1} Y_{i-1, t-1}+b_{i+1} Y_{i+1, t-1}$

\begin{tabular}{|c|c|c|c|c|c|c|c|c|}
\hline Plant & $Y_{i-1, t-1}$ & $\mathrm{SE}^{\mathrm{a}}$ & $P$ value $^{\mathrm{b}}$ & $Y_{i, t-1}$ & SE & $P$ value & $R^{2}$ & $N^{c}$ \\
\hline 1 & 0.003 & 0.00060 & $<0.001$ & 0.009 & 0.00060 & $<0.001$ & 89.1 & 92 \\
\hline 2 & 0.001 & 0.00047 & 0.022 & 0.011 & 0.00047 & $<0.001$ & 94.7 & 92 \\
\hline 3 & & & & 0.014 & 0.00034 & $<0.001$ & 96.1 & 68 \\
\hline 6 & 0.003 & 0.00059 & $<0.001$ & 0.010 & 0.00059 & $<0.001$ & 92.6 & 71 \\
\hline 7 & & & & 0.012 & 0.00034 & $<0.001$ & 94.3 & 77 \\
\hline 11 & & & & 0.012 & 0.00036 & $<0.001$ & 92.2 & 96 \\
\hline 12 & 0.001 & 0.00044 & $<0.001$ & 0.012 & 0.00044 & $<0.001$ & 95.8 & 78 \\
\hline 13 & 0.001 & 0.00058 & $<0.001$ & 0.012 & 0.00058 & $<0.001$ & 93.5 & 104 \\
\hline 16 & 0.002 & 0.00062 & $<0.001$ & 0.009 & 0.00062 & $<0.001$ & 91.2 & 72 \\
\hline 17 & 0.002 & 0.00062 & $<0.001$ & 0.009 & 0.00062 & $<0.001$ & 91.5 & 78 \\
\hline 21 & & & & 0.010 & 0.00053 & $<0.001$ & 79.5 & 99 \\
\hline 22 & 0.002 & 0.00046 & 0.001 & 0.009 & 0.00046 & $<0.001$ & 92.4 & 87 \\
\hline 23 & 0.001 & 0.00052 & 0.025 & 0.010 & 0.00052 & $<0.001$ & 92.6 & 77 \\
\hline 26 & & & & 0.012 & 0.00035 & $<0.001$ & 93.2 & 77 \\
\hline 27 & 0.002 & 0.00042 & $<0.001$ & 0.011 & 0.00042 & $<0.001$ & 95.4 & 76 \\
\hline 31 & & & & 0.014 & 0.00027 & $<0.001$ & 96.3 & 105 \\
\hline 32 & & & & 0.012 & 0.00031 & $<0.001$ & 94.0 & 91 \\
\hline 33 & 0.001 & 0.00038 & $<0.001$ & 0.013 & 0.00038 & $<0.001$ & 97.0 & 71 \\
\hline 36 & 0.002 & 0.00049 & $<0.001$ & 0.012 & 0.00049 & $<0.001$ & 96.7 & 101 \\
\hline 37 & & & & 0.017 & 0.00057 & $<0.001$ & 91.9 & 77 \\
\hline Minimum $^{\mathrm{d}}$ & 0.001 & & & 0.009 & & & & \\
\hline Maximum $^{\mathrm{e}}$ & 0.003 & & & 0.017 & & & & \\
\hline Median $^{f}$ & 0.002 & & & 0.012 & & & & \\
\hline
\end{tabular}

a Standard error of the parameter estimate.

b Level of significance.

c Number of target leaf layers at position $i$.

${ }^{\mathrm{d}}$ Minimum value of the parameter estimate.

e Maximum value of the parameter estimate.

${ }^{\mathrm{f}}$ Median value of parameter estimate. 
disease increase on leaf layer $i$ at time $t$ (Table 3). The range of parameter estimates for the lower leaf layer of the sprayed treatment was, however, the same as that for the unsprayed treatment (Table 1). The parameter estimates for the largest leaf layer for the unsprayed treatment were greater than for the sprayed treatment, indicating a greater contribution of the target leaf layer per unit of disease to disease increase on this leaf layer than with fungicide treatment (Tables 1 and 3). Disease severity on the upper leaf layer made a significant contribution to the model for one plant only, in the sprayed plot at Maasbree (HDS trial) (Table 3 ), but not in the unsprayed field (Table 1).

Sprayed versus unsprayed at LDS (Toldijk) (Tables 2 and 4). The regression coefficients for the target leaf layer were smaller than for the unsprayed treatment, as noted for Maasbree. Unlike Maasbree, regression coefficients for the lower leaf layer were less often significant in the model for the sprayed treatment compared with the unsprayed treatment (Tables 2 and 4). The coefficients were, however, smaller than for the unsprayed treatment (Table 2) as was the case at Maasbree. In other words, per unit of disease the lower leaf layer contributed more to disease increase on the target leaf layer in the unsprayed treatment. Disease severity on the upper leaf layer made a significant contribution for eight of the unsprayed plants (Table 2) but not for the sprayed plants (Table 4).

The contribution of within-row neighboring plants to disease dynamics. In all cases, models fitted were significant with coefficients of determination ranging from 84 to $93 \%$ in the main study areas at Vlodrop and Toldijk 1, and from 75 to $89 \%$ at the additional sites at Koningsbosch, Wehl, and Toldijk 2 (Table 5).

2000 versus 2001. The target plant was more important (per unit of disease) in explaining disease increase on the target plant at both field trials in 2000 (0.318 and 0.312 for Vlodrop and Toldijk1, respectively) than neighboring plants (0.031 and 0.097). In 2001 the effect of neighboring plants was not significant at Vlodrop, but was significant at Toldijk1 with the target plant more important in explaining disease increase $(0.284)$ than the neighboring plants $(0.042)$. There was little difference between the 2 years in the parameter estimates for the target plant in both
Vlodrop and Toldijk; however at Vlodrop, the parameter estimates increased and at Toldijk 1 they decreased (Table 5). The backtransformed estimates of the intercept were close to zero in all cases. In the additional field trials at Koningsbosch (HDS), the neighboring plants in the second year were also not significant in the model (Table 6). The parameter estimate for the target plant at Koningsbosch differed little between the 2 years ( 0.305 in 2000 and 0.329 in 2001). In the additional field trials at Wehl and Toldijk2 (LDS) in 2000, the neighboring plants also made a significant contribution (Wehl, 0.077; Toldijk 2, 0.069), although again of lesser importance than the target plant (Wehl, 0.300; Toldijk 2, 0.342).

HDS versus LDS. A combined analysis was done for trials in the HDS and LDS areas. The target plant was more important $(\mathrm{HDS}=0.329 ; \mathrm{LDS}=0.365)$ than the neighboring plants $(\mathrm{HDS}=$ 0.045 ; LDS $=0.035$ ) (Table 7) in explaining disease increase on the target plant (per unit of disease). The variance accounted for ranged between 82 and $87 \%$.

HDS and LDS combined. Finally all data were pooled (Table 7). The difference between the target and neighboring plants in contributing to disease increase was maintained, as in the individual analyses. The disease increase on a target plant is mostly determined by the target plant itself $(0.321)$ with a lesser contribution from its within-row neighboring plants (0.062). Autoinfection largely determined the increase in disease of CLS on individual sugar beet plants.

\section{DISCUSSION}

In this paper we have analyzed the disease dynamics of CLS on sugar beet using a simple epidemiological model that can be modified to operate at two hierarchical levels, within and between individual plants. Our continuous model is a natural way of representing epidemic dynamics where there are few discontinuities or periodic fluctuations in disease development. In the early stages of an epidemic the infection process is probably discrete as only few spores are available and latent periods probably do not overlap. After a few weeks, when CLS symptoms develop, more

TABLE 5. Disease dynamics between plants: estimates of model parameters (where positive and significant in linear regression) for the contribution of the target and neighboring plants on disease increase of Cercospora leaf spot in sugar beet in the high disease severity (HDS) area at Vlodrop (V) and in the low disease severity (LDS) area at Toldijk1 (T1) in 2000 and 2001

\begin{tabular}{|c|c|c|c|c|c|}
\hline Location & Year & $n^{\mathrm{a}}$ & $R^{2}$ & $\left(Y_{j+1}+Y_{j-1}\right) / 2^{\mathrm{b}}$ & $Y_{j}^{\mathrm{c}}$ \\
\hline \multicolumn{6}{|l|}{ HDS area } \\
\hline V & 2000 & 6,234 & 84.1 & $0.031 \pm 0.008$ & $0.318 \pm 0.008$ \\
\hline $\mathrm{V}$ & 2001 & 5,894 & 92.0 & $\mathrm{~ns}^{\mathrm{d}}$ & $0.373 \pm 0.002$ \\
\hline $\mathrm{T} 1$ & 2000 & 6,038 & 84.4 & $0.097 \pm 0.009$ & $0.312 \pm 0.009$ \\
\hline $\mathrm{T} 1$ & 2001 & 3,780 & 93.0 & $0.042 \pm 0.009$ & $0.284 \pm 0.009$ \\
\hline
\end{tabular}

${ }^{\mathrm{a}} n=$ number of target plants at position $j$.

${ }^{\mathrm{b}}$ Contribution of within-row neighboring plants $\left(\left[Y_{j+1, t-1}+Y_{j-1, t-1}\right] / 2\right)$ to the disease increase of $Y_{j, t} ; 95 \%$ confidence interval follows each estimate.

${ }^{\mathrm{c}}$ Contribution of target plant $Y_{j, t-1}$ at time $t-1$ to the disease increase of $Y_{j, t} ; 95 \%$ confidence interval follows each estimate.

${ }^{\mathrm{d}} \mathrm{ns}=$ not significant $(P=0.05)$.

TABLE 6. Disease dynamics between plants: estimates of model parameters (where positive and significant in linear regression) for the contribution of the target and neighboring plants on disease increase of Cercospora leaf spot in sugar beet in the high disease severity area at Koningsbosch (K) in 2000 and 2001 , and in the low disease severity areas at Wehl (W) and Toldijk2 (T2) in 2000

\begin{tabular}{lcccrc}
\hline Location & Year & $n^{\mathrm{a}}$ & $R^{2}$ & $\left(Y_{j+1}+Y_{j-1}\right) / 2^{\mathrm{b}}$ & $Y_{j}^{\mathrm{c}}$ \\
\hline HDS area & & & & $0.131 \pm 0.010$ & $\mathrm{~ns}^{\mathrm{d}}$ \\
K & 2000 & 6,975 & 86.0 & $0.305 \pm 0.010$ \\
K & 2001 & 5,103 & 89.6 & $0.329 \pm 0.002$ \\
LDS area & & & 75.9 & $0.077 \pm 0.010$ \\
W & 2000 & 4,298 & 86.2 & $0.069 \pm 0.008$ & $0.300 \pm 0.008$ \\
T2 & 2000 & 7,559 & $0.342 \pm 0.008$ \\
\hline
\end{tabular}

a $n=$ number of target plants at position $j$.

${ }^{\mathrm{b}}$ Contribution of within-row neighboring plants $\left(\left[Y_{j+1, t-1}+Y_{j-1, t-1}\right] / 2\right)$ to the disease increase of $Y_{j, t} ; 95 \%$ confidence interval follows each estimate.

${ }^{\mathrm{c}}$ Contribution of target plant $Y_{j, t-1}$ at time $t-1$ to the disease increase of $Y_{j, t} ; 95 \%$ confidence interval follows each estimate.

${ }^{\mathrm{d}} \mathrm{ns}=$ not significant $(P=0.05)$. 
spores are produced with characteristic patterns following a latent period (14) and the infection process is continuous over time with overlapping generations. C. beticola has many infection cycles that can and do occur per growing season (4). Our approach to modeling an epidemic is appropriate when there are several complete infection cycles with overlapping generations over the course of an epidemic $(5,8)$ and the model consistently gave satisfactory fits to the data. The model has proved extremely useful in determining the relative contributions of different leaf layers to disease increase within an individual plant, and of neighboring plants to disease increase on an individual plant; and we suggest it can be used as a calculation tool for this purpose for other polycyclic foliar diseases.

This approach differs from that taken in the hierarchical simulation model of Willocquet and Savary (21) in that it was developed directly for the purpose of analyzing extensive experimental data. No assumptions were made on the ratio of auto- to alloinfection: as noted (21), and in only a few instances have these ratios been measured.

Disease dynamics on individual plants. Applying fungicides lowered mean leaf area diseased from 4 to $1.3 \%$ in the LDS trials at Toldijk. In both treatments, the target leaf layer $i$ was always significant in the model, except for one plant in the unsprayed treatment. Fungicide treatments gave lower parameter estimates for the relative contributions of both the target and lower leaf layers to disease increase, in contrast to the HDS trials. The number of times the lower leaf layer was significantly fewer with the spray treatment, which is also in contrast to the HDS trials. This might be due to differences in varieties or cultural conditions, leading to differences in foliage production and fungicide coverage. The absence of a significant contribution of the upper leaf layer to disease increase when a fungicide was applied, supports the view that rain runoff dispersal of spores (11) was inconsequential under these conditions. We can conclude that with two fungicide applications, the pattern of disease dynamics withinplant was significantly altered.

However, in the LDS trials (Toldijk) we observed a greater influence of the upper leaf layer to disease increase on the target leaf layer in unsprayed conditions than in the HDS trials (Maasbree). This apparent difference in within-plant spread may be due to a range of factors, including inoculum presence and differences in rainfall between the two fields. Both varieties were equally susceptible to CLS, but the structure of the leaf canopy could have had an effect on the splash-dispersal of the pathogen. Pielaat et al. (11) concluded that splash dispersal was concentrated in the upper canopy layers in a crop having a leaf area index constant or increasing with height, as is the case for sugar beet.

Many researchers consider that first, infection by CLS takes place by splashing of conidia from the soil where infected leaf debris serves as the source of inoculum $(7,9)$, although there are some problems in interpretation. The splashing of spores from leaf debris depends on a number of coincident factors; e.g., after a 3-year rotation CLS-diseased leaf debris must be at the soil surface and must be sporulating. The patterns of diseased plants observed early in the season suggests that disease starts on many plants (19). Even in fields with wide crop rotations (1:5), severe
CLS epidemics can occur. Insects and wind are also considered dispersal agents $(2,22)$, and the role of human movements and agricultural machinery is unquantified. The presence of weedhosts and other crops that can maintain $C$. beticola may also influence timing and place of the first infection (4). Another possible infection site for $C$. beticola can be the root, as described by Vereijssen et al. (20). The mechanisms involved are unknown, however, neither epiphytic growth or upward transport of conidia through the xylem have been studied, and the effect of root infection on within-plant disease increase is not known and needs further research.

Overall, CLS was more severe in unsprayed treatment at the HDS site than LDS. Where fungicides were applied the severity was much reduced to similar levels. In the unsprayed plots the target leaf was the most important per unit of disease in contributing to disease increase, whereas in the LDS area, the lower leaf layer and to a lesser extent the upper leaf layer contributed. Under sprayed conditions there was not the same contrast between the two sites. We conclude from this that where disease pressure is high, as in the unsprayed HDS area, the level of autoinfection and disease intensification on an individual leaf is the key driving factor. Where disease pressure is lower, and certainly under sprayed conditions, this influence is less marked.

Disease dynamics between individual plants. In the above study we assumed that visually observed disease increase is due only to inoculum produced within the plant itself. However, Vereijssen et al. (19) have shown that disease severity within-row displays spatial and temporal dependencies, indicating spread of viable conidia to neighboring plants. In this accompanying study we have assessed the contribution of neighboring plants within rows to disease increase using comprehensive field data from five field sites over one or two growing seasons. The relative contribution of neighboring plants to disease increase on a defined plant was assessed. The temporal analysis in this paper builds upon the insight gained from the spatial analysis (19) and incorporates and assesses the importance of neighboring plants to temporal disease increase on an individual plant.

The results in this study confirm the earlier results (19), that disease on a defined plant depends in part on its within-row neighboring plants. Neighboring plants were less important for disease dynamics in the second year trials in 2001 than in 2000. The estimated parameters for allo-infection (from neighboring plants) were smaller or not significant in 2001, indicating more auto-infection than allo-infection. Auto-infection indicates that increase in disease on a defined plant is mainly determined by the disease severity on that plant. Also in this study, auto-infection was more prominent in LDS areas than in HDS areas. In the pooled analysis, disease on target plants was five times more important (per unit of disease) than disease on its neighbors in explaining disease increase, which again indicates the predominant role of auto-infection rather than allo-infection. An interesting question is why the observed differences in parameter estimates between the 2 years occurred at the HDS areas where CLS has been much longer established. There could be several reasons for these differences, including crop rotation, the tillage system used, and local weather conditions. We have no direct evidence for

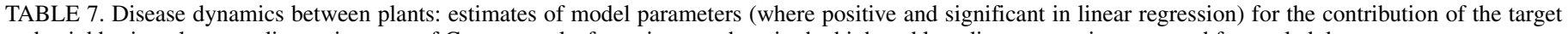
and neighboring plants on disease increase of Cercospora leaf spot in sugar beet in the high and low disease severity areas and for pooled data

\begin{tabular}{|c|c|c|c|c|}
\hline Area $^{\mathrm{a}}$ & $n^{\mathrm{b}}$ & $R^{2}$ & $\left(Y_{j+1}+Y_{j-1}\right) / 2^{\mathrm{c}}$ & $Y_{j}^{\mathrm{d}}$ \\
\hline High severity & 25,325 & 86.5 & $0.045 \pm 0.005$ & $0.329 \pm 0.005$ \\
\hline Low severity & 13,882 & 82.1 & $0.035 \pm 0.006$ & $0.365 \pm 0.006$ \\
\hline Pooled data & 46,506 & 85.3 & $0.062 \pm 0.003$ & $0.321 \pm 0.003$ \\
\hline
\end{tabular}

a Data of all fields in either the high and low disease severity area were pooled for these analyses. For pooled data, all data of both areas were pooled.

${ }^{\mathrm{b}} n=$ number of target plants at position $j$.

${ }^{\mathrm{c}}$ Contribution of within-row neighboring plants $\left(\left[Y_{j+1, t-1}+Y_{j-1, t-1}\right] / 2\right)$ to the disease increase of $Y_{j, t} ; 95 \%$ confidence interval follows each estimate.

${ }^{\mathrm{d}}$ Contribution of target plant $Y_{j, t-1}$ to the disease increase of $Y_{j, t} ; 95 \%$ confidence interval follows each estimate. 
which of these factors were important. However, the fundamental principle remains that neighboring plants were less important in explaining disease increase on a target plant than the target plant itself.

The continuous model explains a high percentage of variance by partitioning the relative contributions of neighboring plants to disease increase. The intercept also indicates that there can be an increase in disease when no disease is present on a plant, e.g., due to external inoculum. In this study the intercept could represent increase in disease due to continuing infection from infected debris, across row neighbors, neighbors more than one plant away, inoculum blown in from other fields, or mechanical spread (humans, machinery, and insects) of inoculum. Backtransformed values of the fitted intercept were consistently close to zero, suggesting that these factors made a very small contribution to the disease increases observed.

We have shown that the spatial contribution of neighboring plants is partly responsible in explaining disease increase on the target plant, as was found by Vereijssen et al. (19). However, disease on the target plant itself (auto-infection) was by far the more important in explaining the disease increase. Similarly, when considering disease increase within an individual plant, auto-infection with respect to a given leaf layer makes a greater contribution to disease increase within an individual plant than allo-infections with respect to the leaf layers below or above the defined layer. Combining both sets of results highlights the limited but important range in spore dispersal and suggests that supervised control of $C$. beticola as proposed by Vereijssen et al. (18), could be further refined by location specific fungicide application to infection foci. In addition (site) specific cultural management practices could be developed, for example plowing under of, or removing crop debris. More generally, this study has confirmed the importance of considering hierarchical scale, and offers a practical approach that complements more complex simulation studies (21) in analyzing disease dynamics.

\section{ACKNOWLEDGMENTS}

We thank F. van den Bosch and S. Pietravalle (IACR-RES, Rothamsted, England) for their hospitality; and S. Pietravalle for writing the GenStat program for fitting the various models, and his patience in trouble-shooting when the analyses started.

\section{LITERATURE CITED}

1. Battilani, P., Beltrami, G., Meriggi, P., Ponti, I., Rossi, A., Rossi, V., Rosso, F., Tugnoli, V., and Zocca, A. 1990. Nuovi indrizzi di difesa anticercosporica. L'Informatore Agrario 46:53-70.

2. Carlson, L. W. 1967. Relation of weather factors to dispersal of conidia of Cercospora beticola (Sacc.). Journal of the American Society of Sugar Beet Technologists 14:319-323.

3. Fitt, B. D. L., McCartney, H. A., and Walklate, P. J. 1989. The role of rain in dispersal of pathogen inoculum. Annu. Rev. Phytopathol. 27:241-270.
4. Frandsen, N. O. 1955. Über den Wirtskreis und die systematische Verwandschaft von Cercospora beticola. Archiv für Mikrobiol. 22:145-174.

5. Jeger, M. J. 1987. Modeling the dynamics of pathogen populations. Pages 91-107 in: Populations of Plant Pathogens: Their Dynamics and Genetics. M. S. Wolfe, and C. E. Caten Eds. Blackwell Scientific Publications, Oxford.

6. Jones, R. K., and Windels, C. E. 1991. A management model for Cercospora leaf spot of sugarbeets. Minnesota Extension Service, AGFO-5643-E.

7. McKay, M. B., and Pool, V. W. 1918. Field studies of Cercospora beticola. Phytopathology 8:119-136.

8. Matko, D., Zupancic, B., and Karba, R. 1992. Simulation and Modeling of Continuous Systems: A Case-Study Approach. Prentice Hall, London.

9. Nagel, C. M. 1938. The longevity of Cercospora beticola in soil. Phytopathology 28:342-350.

10. Pfleiderer, U. E., and Schäufele, W. R. 2000. Pages 147-153 in: Advances in Sugar Beet Research Vol. 2: Cercospora beticola Sacc. Biology, Agronomic Influence and Control Measures in Sugar Beet. Development of a testing method for resistance against Cercospora beticola in sugar beet. M. J. C. Asher, B. Holtschulte, M. Richard Molard, F. Rosso, G. Steinruecken, and R. Beckers, eds. International Institute for Beet Research, Brussels, Belgium.

11. Pielaat, A., van den Bosch, F., Fitt, B. D. L., and Jeger, M. J. 2002. Simulation of vertical spread of plant diseases in a crop canopy by stem extension and splash dispersal. Ecol. Model. 151:195-212.

12. Pundhir, V. S., and Mukhopadhyay, A. N. 1987. Epidemiological studies on Cercospora leaf spot of sugar beet. Plant Pathol. 36:185-191.

13. Rossi, V. 1997. Use of a simulation model "CERCODEP" in the control of Cercospora leaf spot on sugarbeet. Pages 355-359 in: Proc. 60th IIRB Congress. International Institute for Beet Research, Brussels, Belgium.

14. Segarra, J., Jeger, M. J., and Van den Bosch, F. 2001. Epidemic dynamics and patterns of plant diseases. Phytopathology 91:1001-1010.

15. Shane, W. W., and Teng, P. S. 1992. Impact of Cercospora leaf spot on root weight, sugar yield, and purity of Beta vulgaris. Plant Dis. 76:812820.

16. Skaracis, G. N., Ioannidis, P. M., and Ioannidis, P. I. 1996. Integrated management systems against sugarbeet Cercospora leaf spot disease. Pages 45-54 in: Proc. 59th IIRB Congress. International Institute for Beet Research, Brussels, Belgium.

17. Vereijssen, J., Schneider, J. H. M., Termorshuizen, A. J., and Jeger, M. J. 2003. Comparison of two disease assessment keys to assess Cercospora beticola in sugar beet. Crop Prot. 1:201-209.

18. Vereijssen, J., Schneider, J. H. M., and Jeger, M. J. 2007. Supervised control of Cercospora beticola in sugar beet. Crop Prot. 26:19-28.

19. Vereijssen, J., Schneider, J. H. M., Jeger, M. J., and Stein, A. 2007. Spatial pattern of Cercospora leaf spot of sugar beet in long- and recentlyestablished areas. Eur. J. Plant Pathol. 116:187-198.

20. Vereijssen, J., Schneider, J. H. M., and Termorshuizen, A. J. 2004. Possible root infection of Cercospora beticola in sugar beet. Eur. J. Plant Pathol. 110:103-106.

21. Willocquet, L., and Savary, S. 2004. An epidemiological simulation model with three scales of spatial hierarchy. Phytopathology 94:883891.

22. Windels, C. E., Arthur Lamey, H., Hilde, D., Widner, J., and Knudsen, T. 1998. A Cercospora leaf spot model for sugar beet: In practice by an industry. Plant Dis. 82:716-726.

23. Wolf, P. F. J., and Verreet, J. A. 2002. An integrated pest management system in Germany for the control of fungal leaf diseases in sugar beet: The IPM sugar beet model. Plant Dis. 86:336-344. 